\title{
The Kerr-Newman black hole as a rotating Van der Waals gas
}

\author{
F. D. Villalba* \\ Departamento de Física, Universidad de los Andes, \\ Apartado Aéreo 4976, Bogotá, Distrito Capital, Colombia \\ A. F. Vargas ${ }^{\dagger}$ \\ School of Physics, University of Melbourne, Parkville VIC 3010, Australia \\ E. Contreras ${ }^{\dagger}$ \\ Departamento de Fúsica, Colegio de Ciencias e Ingeriería, \\ Universidad San Francisco de Quito, Quito, Ecuador \\ Pedro Bargueño§ \\ Departamento de Física Aplicada, Universidad de Alicante, \\ Campus de San Vicente del Raspeig, E-03690 Alicante, Spain
}

\begin{abstract}
In this work we employ the horizon approach for the thermodynamics of black holes, together with holographic equipartition, to show that the Kerr-Newman black hole can be considered as a rotating Van der Waals gas. Our results are interpreted in light of a simple mechanical and geometrical model, which is amenable to emerge from the space-time atom perspective.
\end{abstract}

\section{INTRODUCTION}

The incorporation of a pressure and its conjugate volume [1-4] into the scheme of black hole (BH) thermodynamics provoked a renewed interest in this field of study. Within this extended framework, the pressure follows from allowing the cosmological constant, $\lambda$, to be dynamical [5]. When considering AdS-like solutions, it has been shown that their thermodynamic phase structure coincides with that of a Van der Waals (VdW) gas (see [6] for an extended review). This inspires the question for a possible "microscopic" interpretation of the $\mathrm{VdW}$ like behavior of the $\mathrm{AdS} \mathrm{BH}$ solution. Work has been done in this direction ranging from approaching the above connection as the emergent result of certain space-time atoms statistics $[7,8], \mathrm{BH}$ molecules $[9,10]$, to other approaches $[11,12]$ based on Ruppeiner geometry [13]. By emergent we mean the appearance of certain degrees of freedom (dof), quantum gravitational or otherwise, whose microscopical dynamics manifests as $\mathrm{BH}$ thermodynamics. In this sense, the quest for the true nature of $\mathrm{BH}$ dof, which has been addressed from various corners of theoretical physics including string theory [14], loop quantum gravity [15], and AdS/CFT [16], among others, could be approached from a new angle exploiting this unsuspected connection.

From the pioneering work of Bekenstein [17] and the subsequent Bekenstein-Mukhanov quantum $\mathrm{BH}$ models [18], the idea of a quantized horizon area,

\footnotetext{
* fd.villalba10@uniandes.edu.co

† afvargas@student.unimelb.edu.au

$\ddagger$ econtreras@usfq.edu.ec

$\S$ pedro.bargueno@ua.es (corresponding author)
}

composed of a natural number of Planck areas $\left(l_{p}^{2}\right)$ that locally encode bits of information, has become widespread. Although originally the bits served as a heuristic explanation, approaches such as the coined holographic principle [19] have tried to rewrite $\mathrm{BH}$ thermodynamics in terms of these dof.

With this context in mind, tiling the horizon has become a tool frequently used to construct microscopical models starting from these entities [20]. Among these models, Padmanabhan's holographic energy equipartition [7] considers that the horizon can be tessellated with $N=A / l_{p}^{2}$ units, where $A$ is the area of the $\mathrm{BH}$ horizon. Intringuingly, these $N$ constituents, usually coined space-time atoms, satisfy an energy equipartition similar to what is found in usual classical models of matter (see Ref. [21] for a recent account on $\mathrm{BH}$ dof within the extended thermodynamic context). Recently, an exact $\mathrm{VdW}$ equation of state (EoS) has been derived from certain non-rotating $\mathrm{BH}$ solutions from basic statistical considerations [8] by using the space-time atoms approach in the context of the extended thermodynamic framework. In particular, a microscopical interpretation for the thermodynamical volume, together with a gravity-induced modified counting of the holographic dof in different dimensions has been proposed in this Reference.

Following these previous works, it is our purpose here to obtain an EoS for the Kerr-Newman (KN) BH, which could be interpreted from a statistical mechanical approach in the sense of Ref [8]. We would like to point out that previous works in Extended Phase Space (EPS) and Horizon Thermodynamics (HT) have found an EoS for this $\mathrm{BH}$ solution, yet to this moment no microscopical interpretation has been presented.

This work is organized as follows: in Section II, the EPS, HT and the holographic equipartition approaches are briefly reviewed and then used to rederive the AdS- 
Reissner Nördstrom (RN) and AdS-KN solutions in subsections II A and II B. Section III is devoted to obtaining a VdW type EoS for the KN BH in the light of both the HT and holographic equipartition approaches, whose features and implications are discussed. Final comments and future work are left to Section IV.

\section{BLACK HOLE EQUATIONS OF STATE}

This section is devoted to summarize the main ideas leading to the concept of BH EoS using both the EPS and HT approaches. Emphasis will be given to the construction and interpretation of the EoS for the AdS-RN BH.

\section{A. Extended Phase Space}

In this section we review the EPS approach [4, 22-28] or Black Hole Chemistry [6], as it has been usually coined. This particular approach answers the question of "where is the PdV term in the first law of BH mechanics?" by considering a $\mathrm{BH}$ spacetime with an AdS background, for which the cosmological constant $\lambda$ is taken as a dynamical value. By perturbing the spacetime with cosmological constant $\lambda$ to a spacetime with $\lambda+d \lambda$, the first law can be written as $d M=T d S+V_{t} d P_{\lambda}+\phi d Q+\ldots$, where $V_{t}$ is considered a thermodynamical volume conjugate to the pressure $P_{\lambda}$, given by $P_{\lambda}=-\frac{\lambda}{8 \pi}$. An important consequence of this extended first law, is the new identification of the mass $M$ with the thermodynamical enthalpy $H$ [4], instead of the internal energy $U$. As such, the mass $M$ is a function of the pressure $P_{\lambda}$ and entropy $S$, plus a number of conserved charges $Q_{i}, i$. e. $M=M\left(P_{\lambda}, S, Q_{i}\right)$ with the temperature $T$ and thermodynamical volume $V_{t}$ as conjugate variables, given by $T=\left(\frac{\partial M}{\partial S}\right)_{P_{\lambda}}$ and $V_{t}=\left(\frac{\partial M}{\partial P_{\lambda}}\right)_{S}$, as expected from the usual thermodynamical theory. For simplicity in our notation, in what follows we denote $V_{t}$ simply as $V$, understanding that this volume is thermodynamical in nature.

In addition to the EPS approach, the so-called holographic energy equipartition [7] plays a fundamental role in order to shed light on the dof emerging in the thermodynamical description of BHs. Following [7], the Komar energy can be written on the horizon as $E_{K}=\frac{1}{2} N T$, where $T$ stands for the local Hawking temperature measured by an observer at rest in this spacetime and $N=$ $d^{2} x \sqrt{\sigma} l_{p}^{-2}$ stands for the microscopic dof attributed to an element of area $A=d^{2} x \sqrt{\sigma}$. In particular, for an AdS-RN BH, it can be shown [8] that the corresponding EoS can be written exactly as

$$
P_{\lambda}=\frac{\bar{N} T}{V}-\frac{1}{2 \pi} \frac{\bar{N}^{2}}{V^{2}}+\frac{2 Q^{2}}{\pi} \frac{\bar{N}^{4}}{V^{4}},
$$

where the thermodynamic volume, $V$, turns out to be Euclidean, $V=\frac{4}{3} \pi r_{+}^{3}, T$ is the corresponding Hawking temperature and $Q$ is the electric charge. Importantly, $P_{\lambda}$ is considered as a pressure due to the cosmological constant and $\bar{N}=\frac{N}{6}$ are the dof compatible with holographic equipartition. Therefore, as it can be shown by inspection, the meaning of the rhs of Eq. (1) remains clear: we have kinetic, interaction and second virial terms. Thus, a microscopic model based on these ingredients can be constructed in order to show how thermodynamic properties of AdS-RN BHs can be derived from a statistical model [8].

The case for a rotating $\mathrm{BH}$ is, as usual, more complicated that its non-rotating counterpart. Let us consider an AdS-KN BH whose $D=4$ metric takes the following form in Boyer-Lindquist coordinates:

$$
\begin{aligned}
d s^{2} & =-\frac{\Delta_{r}}{\rho^{2}}\left(d t-\frac{a \sin ^{2} \theta}{\Xi} d \phi\right)^{2}+\frac{\rho^{2}}{\Delta_{r}} d r^{2}+\frac{\rho^{2}}{\Delta_{\theta}} d \theta^{2} \\
& +\frac{\Delta_{\theta} \sin ^{2} \theta}{\rho^{2}}\left[a d t-\frac{\left(r^{2}+a^{2}\right)}{\Xi} d \phi\right]^{2}
\end{aligned}
$$

where

and

$$
\begin{aligned}
\rho^{2} & =r^{2}+a^{2} \cos ^{2} \theta, \\
\Xi & =1-\frac{a^{2}}{l^{2}}, \\
\Delta_{\theta} & =1-\frac{a^{2}}{l^{2}} \cos ^{2} \theta
\end{aligned}
$$

$$
\Delta_{r}=\left(r^{2}+a^{2}\right)\left(1+\frac{r^{2}}{l^{2}}\right)-\Xi^{2}\left(2 M r-Q^{2}\right) .
$$

The horizon radius $r_{+}$is determined by the largest root of $\Delta_{r}=0$. Following the procedure presented for the AdS-RN BH, we can obtain all the thermodynamic variables for the system. For example, the thermodynamical volume is given by

$$
V=\frac{2 \pi}{3}\left(\frac{\left(r_{+}^{2}+a^{2}\right)\left(2 r_{+}^{2} l^{2}+a^{2} l^{2}-r_{+}^{2} a^{2}\right)+l^{2} \Xi^{2} Q^{2} a^{2}}{l^{2} \Xi^{2} r_{+}}\right) .
$$

Therefore, although it is possible to obtain an explicit thermodynamical volume within the EPS approach, it can not be used to construct an EoS in terms of the pressure. As seen in Eq. (7), the thermodynamical volume of the AdS-KN BH is a complicated function of the AdS radius, $l^{2}$, and therefore of the pressure, given by $P=3 / 8 \pi l^{2}$ as in the non-rotating case. Even more, although the function given by Eq. (7) can be inverted, that is $V(P) \rightarrow P(V)$, a readable and easily interpretable EoS for this particular BH, within the presented approach, has not been found.

In this sense, there is no possibility of employing holographic equipartition within the EPS approach in order to shed some light into a possible statistical description for rotating $\mathrm{BHs}$, in contrast with the non-rotating case, as previously commented. However, a different way to derive $\mathrm{BHs}$ EoS in order to link them with holographic 
equipartition techniques can be employed. The next section is devoted to give the reader a brief description of this alternative route, which is known as horizon thermodynamics.

\section{B. Horizon thermodynamics}

The origin of HT was the realization [29] that the Einstein equations on the horizon of spherically symmetric spacetimes can be interpreted as a thermodynamical identity. This relevant observation has been extended to other cases corresponding to different gravitational theories and symmetries (for a review see [7, 30]), and also to the study of the thermodynamics of null surfaces [31]. The basic idea of HT is to make the identification $P_{\text {tot }}=T_{r}^{r}$, being $T_{\mu \nu}$ the energy-momentum tensor of the complete matter sector (including a possible cosmological constant) evaluated at the horizon. Then, under the assumption of an Euclidean geometric volume for the $\mathrm{BH}$, the radial Einstein equation can be interpreted as an EoS for spherically symmetric AdS BHs in the form $P_{\text {tot }}=P_{\text {tot }}(T, V)[32]$. Interestingly, this EoS does not depend on the specific form of $g_{t t}\left(=g^{r r}\right)$. Even more, the authors of Ref. [32] derive the first law of HT in the form $d E=T d S-P_{t o t} d V$, where $E$ is an energy associated with the $\mathrm{BH}$ whose meaning is discussed in that Reference. In addition, the horizon enthalpy and Gibbs energy are defined according to the standard prescriptions $G=E-T S+P V$ and $H=G+T S$.

Although there are several differences between the EPS and HT approaches [32], here we would like to remark one of them which is of relevance for this work, namely: for the AdS-RN BH, the volume is assumed to be the Euclidean geometrical volume, being independent on the matter sector in HT, in contrast with the EPS approach, in which the volume is conjugate to the pressure given by the cosmological constant and depends on the matter content of the theory. Finally, we recall that only in vacuum and for $P_{\text {tot }}>0$, both the EPS and the HT approaches yield the same kind of thermodynamic behaviours and phase transitions [32].

In order to compare HT with EPS, let us first consider the AdS-RN case. For this system, following [32], it is easy to see that the EoS is

$$
P_{t o t}=\frac{T}{2 r_{+}}-\frac{1}{8 \pi r_{+}^{2}},
$$

where $V=\frac{4 \pi r_{+}^{3}}{3}$ and

$$
P_{t o t}=P_{m}+P_{\lambda}
$$

with

$$
P_{m}=T_{r}^{r}=-\frac{Q^{2}}{8 \pi r_{+}^{4}}
$$

is the electromagnetic radiation pressure exerted on the horizon due to the matter terms of the Einstein equations. Finally, we note that, although Eqs. (1) and (8) coincide, the thermodynamical behaviour which they describe is different, as pointed out in [32].

For the rotating case, let us consider a pure Kerr geometry $(l \rightarrow \infty$ and $Q=0)$. Here, in analogy with the static case, after using the radial Einstein equation the corresponding first law is obtained in the form [32]

$$
d E=T d S+\Omega d J-\sigma d A
$$

where

$$
E=\frac{r_{+}^{2}+a^{2}}{2 r_{+}}
$$

is the horizon energy [32], $J=E a$ is the $\mathrm{BH}$ angular momentum,

$$
\Omega=\frac{a}{r_{+}^{2}+a^{2}},
$$

is the horizon angular velocity,

$$
T=\frac{r_{+}-M}{2 \pi\left(r_{+}^{2}+a^{2}\right)}
$$

is the $\mathrm{BH}$ temperature, $S=\pi\left(r_{+}^{2}+a^{2}\right)=\frac{A}{4}$ its entropy with $A$ the horizon area and

$$
\sigma=\frac{\left.\rho_{+}^{4} T_{r}^{r}\right|_{r_{+}}}{2 r_{+}\left(r_{+}^{2}+a^{2}\right)},
$$

is the so-called surface tension [32].

To study the EoS for the rotating $\mathrm{BH}$, the authors of Ref. [32] rewrite the last term in Eq. (11) as

$$
\sigma d A=P d V
$$

by noting that this equality is only possible when $d V \sim d A$. Specifically, the three different cases considered in [32] are:

(i) After imposing

$$
V=\frac{4 \pi}{3}\left(\frac{A}{4 \pi}\right)^{3 / 2},
$$

the pressure term is written as

$$
P=\frac{\sigma A}{2 \pi}\left(\frac{4 \pi}{A}\right)^{3 / 2}
$$

and the corresponding EoS can be written as

$$
P=\frac{T}{2 \sqrt{r_{+}^{2}+a^{2}}}+\frac{a^{2}-r_{+}^{2}}{8 \pi r_{+}\left(r_{+}^{2}+a^{2}\right)^{3 / 2}},
$$


where $r_{+}$and $a$ are implicit functions of $V$ and $J$.

(ii) After imposing the geometric volume

$$
V=\frac{r_{+} A}{3}
$$

the pressure is written as

$$
P=\frac{6 \sigma\left(r_{+}^{2}+a^{2}\right)}{r_{+}\left(3 r_{+}^{2}+5 a^{2}\right)},
$$

and the EoS reads

$$
P=\frac{3}{2} \frac{T\left(r_{+}^{2}+a^{2}\right)}{r_{+}\left(3 r_{+}^{2}+5 a^{2}\right)}+\frac{3}{8 \pi} \frac{a^{2}-r_{+}^{2}}{r_{+}^{2}\left(3 r_{+}^{2}+5 a^{2}\right)},
$$

where $r_{+}$and $a$ are implicit functions of $V$ and $J$ as in the previous case.

And (iii), if the thermodynamic volume coming from EPS considerations [33] is imposed, $i$. e.

$$
V=\frac{r_{+} A}{3}\left(1+\frac{a^{2}}{2 r_{+}^{2}}\right),
$$

we obtain

$$
P=\frac{12 \sigma r_{+}\left(r_{+}^{2}+a^{2}\right)}{6 r_{+}^{4}+9 r_{+}^{2} a^{2}+a^{4}}
$$

and

$$
P=\frac{3 T\left(r_{+}^{2}+a^{2}\right)}{6 r_{+}^{4}+9 r_{+}^{2} a^{2}+a^{4}}+\frac{3}{4 \pi} \frac{a^{2}-r_{+}^{2}}{6 r_{+}^{4}+9 r_{+}^{2} a^{2}+a^{4}} .
$$

Therefore, after having a look at Eqs. (19), (22) and (25) we conclude that, although this approach is, without any doubt, of great interest, we miss a link with holographic equipartition in order to show if the emergence of some dof, as in the static case, could shed light on a possible microscopical description for rotating BHs. The look for this link, together with a transparent writing of the corresponding EoS, in the lines of Eq. (1), is the purpose of the following section.

\section{THE KERR-NEWMAN BLACK HOLE AS A ROTATING VAN DER WAALS GAS}

Intuitively one is tempted to assign $N=A$ dof to the horizon of a rotating $\mathrm{BH}$, in analogy with the static case. In order to do this formally, holographic equipartition has to be considered in the stationary case. The problem is that, for a stationary spacetime, the situation is more subtle because of the ambiguity of the choice for the Killing vector in the calculation of the Komar energy. By extending the considerations of the static case, one can simply start by supposing that the horizon in the stationary case also has a number of degrees of freedom proportional to its area. The authors of Ref. [34] have followed such an approach for specific 2-surfaces (screens); in addition, they supplemented such assumption with a prescription for the temperature of the screens in terms of a canonical timelike Killing vector, idea inspired by Verlinde's proposal for emergent gravity [35], which removes the aforementioned ambiguity. With these ingredients, they have obtained the Komar energy associated to the considered 2-surfaces in a KN spacetimes, which leads to a recovery of Einstein equations within such context. In the case of the horizon, this Reference shows that the corresponding Komar energy coincides with the reduced mass of the $\mathrm{BH}$ :

$$
E_{K}=\sqrt{M^{2}-a^{2}-Q^{2}}
$$

Although [34] recovers the Komar energy for general screens based on the holographic equipartition and the temperature prescription in terms of a timelike Killing vector, a simpler analysis can illustrate this point when the screen corresponds to the horizon, given that the temperature of the horizon is defined in a straightforward way through its surface gravity.

Let us recall that the Hawking temperature for a $\mathrm{KN} \mathrm{BH}$ is

$$
T=\frac{r_{+}-M}{2 \pi\left(r_{+}^{2}+a^{2}\right)}
$$

with $r_{+}=M+\sqrt{M^{2}-a^{2}-Q^{2}}$. Therefore, using Eqs. (26) and (27) it follows directly that

$$
E_{K}=\frac{1}{2} N T
$$

is satisfied whenever $N=A=4 \pi\left(r_{+}^{2}+a^{2}\right)$. Thus, Eq. (28) shows that the Komar energy of the horizon is already consistent with the appearance of certain dof, as dictated by holographic equipartition. With these ideas in mind, in what follows we will consider as a basic assumption that the KN BH horizon has a specific number of particles associated.

In order to obtain an EoS for these $N$ particles, we can either use the Smarr law

$$
M=2 T S+2 \Omega J+\phi Q,
$$

where

$$
\phi=\frac{Q r_{+}}{r_{+}^{2}+a^{2}},
$$

is the electric potential at the horizon, or use the horizon condition, which reads

$$
\Delta=r_{+}^{2}-2 M r_{+}+a^{2}+Q^{2}=0 .
$$

In any of these two cases it is enough to express the mass $M$ as a function of the temperature $T$ as

$$
M=r_{+}-2 \pi T\left(r_{+}^{2}+a^{2}\right),
$$


and then manipulate Eq. (29) or Eq. (31) by introducing some appropriate definitions. As the second way is even shorter, let us choose it. Then, in order to obtain the EoS we demand that

$$
\frac{\Delta}{8 \pi r_{+}^{4}}=0
$$

At first sight this seem to be an arbitrary choice (and in fact it is) but we will see that it is intimately linked with the choice of the geometric volume as that which enters in the BH EoS.

For a KN BH, Eq. (33) reads

$$
\frac{1}{8 \pi r_{+}^{2}}-\frac{M}{4 \pi r_{+}^{3}}+\frac{a^{2}}{8 \pi r_{+}^{4}}+\frac{Q^{2}}{8 \pi r_{+}^{4}}=0
$$

As previously commented, let us introduce the geometric volume

$$
V=\frac{4 \pi r_{+}^{3}}{3}
$$

Within this choice, Eq. (34) can be written as

$$
P_{\sigma}+P_{m}+P_{a}=\frac{\bar{N} T}{V}
$$

At this point, a couple of comments are in order. First, the rhs of Eq. (36) refers to $\bar{N}=\frac{N}{6}$ particles living inside a spherical container of radius $r_{+}$at temperature $T$. Second, the lhs is divided in three contributions. The first one, $P_{\sigma}$, is the horizon energy density [32]. This term is expressed as

$$
P_{\sigma}=\frac{k_{g}}{8 \pi}
$$

where $k_{g}=\frac{1}{r_{+}^{2}}$ is the Gaussian curvature of the twosphere. The second term, $P_{m}$, is the radiation pressure on the sphere,

$$
P_{m}=-\frac{Q^{2}}{8 \pi r_{+}^{4}}
$$

and the third term,

$$
P_{a}=-\frac{a^{2}}{8 \pi r_{+}^{4}}
$$

can be interpreted as a pressure term due to the rotation of the $\mathrm{BH}$. And third, a $\mathrm{KN} \mathrm{BH}$ could be thought to be an ideal gas of $\bar{N}=\frac{A}{6}$ dof enclosed inside a spherical container of radius $r_{+}$in equilibrium with a total pressure given by

$$
P_{\text {tot }}=P_{\sigma}+P_{m}+P_{a}
$$

at the corresponding Hawking temperature. However, a has to be written as an implicit function of $V$ and $J$, as mentioned in previous cases. Then, using that

we arrive to

$$
J=\frac{a}{2 r_{+}}\left(r_{+}^{2}+a^{2}\right),
$$

$$
P_{\sigma}+P_{m}=\frac{\bar{N} T}{V}+\frac{8 \pi}{9} \frac{J^{2}}{\bar{N}^{2} r_{+}^{2}} .
$$

For comparison with the RN case, let us write the two EoS in a similar form: where the subindex 0,1 refers to the dof corresponding to $\mathrm{RN}$ and KN BHs given by holographic equipartition, respectively, $i$. e. $N_{0}=4 \pi r_{+, \mathrm{RN}}^{2}$ and $N_{1}=4 \pi\left(r_{+, \mathrm{KN}}^{2}+a^{2}\right)$.
At first sight the only novelty is the $\frac{8 \pi}{9} \frac{J^{2}}{N_{1}^{2} r_{+}^{2}}$ term. However, when these equations are written explicitly in terms of the dof we are led to

$$
\begin{aligned}
& (\mathrm{RN}): 0=\frac{\bar{N}_{0} T}{V}-\frac{1}{2 \pi} \frac{\bar{N}_{0}^{2}}{V^{2}}+\frac{2 Q^{2}}{\pi} \frac{\bar{N}_{0}^{4}}{V^{4}} \\
& (\mathrm{KN}): 0=\frac{\bar{N}_{1} T}{V}+\frac{8 \pi}{9} \frac{J^{2}}{\bar{N}_{1}^{2} r_{+}^{2}}-\frac{1}{2 \pi} \frac{\left(\bar{N}_{1}-2 \pi a^{2} / 3\right)^{2}}{V^{2}}+\frac{2 Q^{2}}{\pi} \frac{\left(\bar{N}_{1}-2 \pi a^{2} / 3\right)^{4}}{V^{4}}
\end{aligned}
$$


Now we write the EoS for the KN BH as

$$
\tilde{P}_{\sigma}+P_{m}=\frac{\bar{N}_{1} T}{V},
$$

where

$$
\tilde{P}_{\sigma}=P_{\sigma}\left[1-\left(\frac{8 \pi J}{3 \bar{N}_{1}}\right)^{2}\right]=P_{\sigma}+P_{r o t},
$$

is a modified horizon energy density which incorporates the effects of the rotation. Surprisingly, this pressure given by Eq. (47) can be derived from the following simple mechanical model as follows.

Let us consider a spherical container of radius $r_{+}$which is rotating with an angular velocity given by $\Omega$. The rotational kinetic energy is given by $E_{\text {rot }}=\frac{1}{2} I \Omega^{2}$, where $I$ is the moment of inertia of the sphere which can be expressed as $I=J / \Omega$, being $J$ the angular momentum (both with respect to an axis which goes through the center of the sphere). For a $\mathrm{KN} \mathrm{BH}$ we know that $\Omega=\frac{2 \pi}{3} \frac{a}{\bar{N}_{1}}$ and $J=\frac{3}{4 \pi r_{+}} a \bar{N}_{1}$. Then, we have that $E_{\text {rot }}=\frac{a^{2}}{4 r_{+}}$. Finally, we note that the pressure exerted on the surface due to the rotation is $P_{\text {rot }}=\frac{E_{r o t}}{V}$ getting $P_{r o t}=\frac{3}{2} \frac{a^{2}}{8 \pi r_{+}^{4}}=\frac{3}{2} \frac{8 \pi}{9} \frac{J^{2}}{N_{1}^{2} r_{+}^{2}}$, which corresponds, up to a factor of $3 / 2$, to the second term of Eq. (47). Interestingly, this simple calculation for the rotational energy coincides with that employed within a dynamical version of the first law [36].

Within this model we emphasize that $P_{m}$ can not be interpreted only as a second virial $\mathrm{VdW}$ term (in contrast with the RN case) because, as shown in Eq. (45), the real dof, $\bar{N}_{1}$, appear in a complicated form. Therefore, the radiation pressure has to be considered as provided by an "external" agent. Interestingly, Eq. (46) can be written as as (in the rest of the manuscript, the dof $\bar{N}_{1}$ will be denoted by $\bar{N}$ without reference to any subscript)

$$
\begin{aligned}
P_{m} & =\frac{\bar{N} T}{V}-\frac{1}{2 \pi} \frac{\bar{N}^{2}}{V^{2}}-P_{r o t}-\frac{P_{r o t}^{2}}{P_{\sigma}}+24 \bar{N} P_{r o t} P_{\sigma} \\
& =\frac{\bar{N} T}{V}-\frac{1}{2 \pi} \frac{\bar{N}}{V^{2}}+P_{r o t}\left(1+\frac{P_{r o t}}{P_{\sigma}}\right) .
\end{aligned}
$$

Note that one can write $1+\frac{P_{\text {rot }}}{P_{\sigma}}=1+\frac{a^{2}}{r_{+}^{2}}=\frac{2 M}{r_{+}}$. Then, we have that $1 \leq 1+\frac{P_{\text {rot }}}{P_{\sigma}} \leq 2$, where the lower value corresponds to the non-rotating case $(\mathrm{RN} \mathrm{BH})$ and the larger value to the extremal KN BH. Therefore, Eq. (48) reads

$$
P_{m}=\frac{\bar{N} T}{V}-\frac{1}{2 \pi} \frac{\bar{N}^{2}}{V^{2}}+K P_{r o t},
$$

where $1 \leq K \leq 2$. Note that $K=1$ implies $P_{\text {rot }}=0$ while $K=2$ corresponds to the extremal $\mathrm{BH}$.

Even more, if we define the distortion parameter of the horizon, $\beta$, following Smarr [37], as

$$
\beta \equiv a\left(r_{+}^{2}+a^{2}\right)^{-1 / 2},
$$

we arrive to

$$
P_{m}+\frac{P_{r o t}}{\beta^{2}-1}=\frac{\bar{N} T}{V}-\frac{1}{2 \pi} \frac{\bar{N}^{2}}{V^{2}} .
$$

Not that, on one hand, $\beta=0$ corresponds to a nonrotating $\mathrm{BH}$. Therefore, $\beta=0$ implies $a=0$ and, then, $P_{\text {rot }}=0$. On the other hand, an extremal BH corresponds to $\beta=1 / \sqrt{2}$. In addition, we remark that the distortion parameter coincides with the eccentricity of the oblate ellipsoid when describing the event horizon in terms of the Cartesian coordinates of the Kerr-Schild line element [38].

\section{DISCUSSION AND FINAL REMARKS}

The main purpose of this work was to obtain an equation of state for the Kerr-Newman black hole such that (i) it can be easily derived and (ii) the physical meaning of their terms was physically transparent. After briefly reviewing both the extended phase and the horizon thermodynamics approaches for the rotating and charged cases, we arrived to the following equation of state:

$$
P_{m}+\frac{P_{\text {rot }}}{\beta^{2}-1}=\frac{\bar{N} T}{V}-\frac{1}{2 \pi} \frac{\bar{N}^{2}}{V^{2}} .
$$

The model this equation describes consists roughly in introducing $\bar{N}$ particles inside a rotating spherical container of radius $r_{+}$at equilibrium at the Hawking temperature. In this equation, $\beta$ is the distortion parameter of the horizon, $P_{m}$ is the radiation pressure exerted on the surface of the container and $P_{\text {rot }}$ corresponds to a pressure due to the rotation of the hole. As in the nonrotating case, $\bar{N}=\frac{A}{6}=\frac{N_{1}}{6}=\frac{4 \pi\left(r_{+}^{2}+a^{2}\right)}{6}$ can be understood as the holographic degrees of freedom.

At this point, a couple of comments are in order. First, we note that, in order to obtain the equation of state here presented for the Kerr-Newman black hole, one can also start from the radial Einstein equation evaluated on the horizon as in Eq. (15) of [32], which reads $\left.8 \pi T_{r}^{r}\right|_{r_{+}}=\left.G_{r}^{r}\right|_{r_{+}}=\frac{a^{2}-r_{+}^{2}+r_{+} \Delta^{\prime}\left(r_{+}\right)}{\rho_{+}^{4}}$, where a prime de- 
notes derivative with respect to the $r$ variable. By noting that $\left.T_{r}^{r}\right|_{r_{+}}=-\frac{Q^{2}}{8 \pi \rho_{+}^{4}}$, the dependence on $\cos \theta$ disappears and, after introducing the geometrical volume, identifying $T=\frac{\Delta^{\prime}\left(r_{+}\right)}{4 \pi\left(r_{+}^{2}+a^{2}\right)}$ and using the holographic equipartition, the equation of state here studied is recovered. Second, an important point to be remembered is that the reduced mass for the Kerr-Newman black hole, $E_{K}$, when computed at the horizon, satisfies a holographic equipartition rule as $E_{K}=\frac{1}{2} N T$, which shows the emergence of the degrees of freedom appearing in the equation of state.

In a previous work [8], these degrees of freedom where used to show how, under certain circumstances, a statistical treatment could reproduce the Van der Waals like equation of state. The gravitational degrees of freedom were reimagined as particles in a container that experienced a contact interaction with the walls. In this dual system, the canonical ensemble partition function sucessfully reproduced the equation of state and the Bekenstein entropy if two heuristic constraints where met. Namely: a modified state counting and a relationship between the de Broglie and specific volumes of the constituents of the dual gas, coined the stacking condition. Since Eq. (51) is a Van der Waals like equation with a modified pressure, it would be interesting to extend the model presented in [8] to account for the new pressures that appear on the left hand side of Eq. (51) via the dual system approach. This extension is left for future work.

In addition, it is worth mentioning that the strategy here developed can be straightforwardly applied to other models such as the well known Kerr-Taub-NUT solution, for example. Even more, it can be applied to generic rotating solutions, as those obtained by the Newman-Janis algorithm, to some extent. Of course, the main issue with generic solutions is that the mass/charge/angular momentum can not be identified unless some particular static seed is specified. We leave these and other problems for future work.

\section{ACKNOWLEDGEMENTS}

P. B. dedicates this work to Anaís, Lucía, Inés and Ana for continuous support. P. B. is funded by the Beatriz Galindo contract BEAGAL 18/00207 (Spain). F. V. acknowledges funding by the Department of Physics of Universidad de los Andes. A.V is supported by a Melbourne Research Scholarship and the N.D. Goldsworthy Scholarship.
[1] S. Wang, S.-Q. Wu, F. Xie, and L. Dan, Chin. Phys. Lett. 23, 1096 (2006).

[2] Y. Sekiwa, Phys. Rev. D 73, 084009 (2006).

[3] E. A. Larranaga Rubio, arXiv:0711.0012 [gr-qc].

[4] D. Kastor, S. Ray and J. Traschen, Class. Quantum Grav. 26, 125020 (2009).

[5] M. Henneaux and C. Teitelboim, Phys. Lett. B, 143, 415 (1984); M. Henneaux and C. Teitelboim, Phys. Lett. B, 222, 195 (1989); C. Teitelboim, Phys. Lett. B, 158, 293 (1985).

[6] D. Kubiznak, R. B. Mann and M. Teo, Class. Quantum Grav. 34, 063001 (2017).

[7] T. Padmanabhan, Mod. Phys. Lett. A 25, 1129 (2010).

[8] A. F. Vargas, E. Contreras and P. Bargueño, Gen. Rel. Grav. 50, 117 (2018).

[9] S.-W. Wei and Y.-X Liu, Phys. Rev. Lett. 115, 111302 (2015); Erratum, Phys. Rev. Lett. 116, 169903 (2016).

[10] Y. -G Miao and Z. -M. Xu, Phys. Rev. D 98, 044001 (2018).

[11] M. K. Zangeneh, A. Dehyadegari, A. Sheykhi, and R. B. Mann, Phys. Rev. D 97, 084054 (2018).

[12] S.-W. Wei, Y.-X. Liu, and R. B. Mann, Phys. Rev. Lett. 123, 071103 (2019).

[13] G. Ruppeiner, Rev. Mod. Phys. 67, 605 (1995); Erratum, Rev. Mod. Phys. 68, 313(E) (1996).

[14] A. Strominger and C. Vafa, Phys. Lett. B 379, 99 (1996); S. D. Mathure, Fortschr. Phys. 53, 793 (2005); A. Sen, Gen. Relativ. Gravit. 46, 1711 (2014).

[15] A. Ashtekar, J. Baez, A. Corichi, and K. Krasnov, Phys. Rev. Lett. 80, 904 (1998); A. Ghosh, K. Noui, and A. Perez, Phys. Rev. D 89, 084069 (2014); A. Perez, Rep. Prog. Phys. 80, 126901 (2017).
[16] J. M. Maldacena, Theor. Phys. 38, 1113 (1999); S. S. Gubser, I. R. Klebanov, and A. M. Polyakov, Phys. Lett. B 428, 105 (1998); E. Witten, Adv. Theor. Math. Phys. 2, 253 (1998).

[17] J. D. Bekenstein, Phys. Rev. D 9, 3292 (1974).

[18] J. D. Bekenstein and V. F. Mukhanov, Phys. Lett. B 360, 7 (1995).

[19] G. 't Hooft, in Salam Festschrifft, edited by A. Aly, J. Ellis, and S. Randjbar Daemi (World Scientific, Singapore, 1993); L. Susskind, J. Math. Phys. (N.Y.) 36, 6377 (1995).

[20] S. Hod, Phys. Rev. Lett. 81, 4293 (1998); I. B. Khriplovich, Phys. Lett. B 431, 19 (1998); G. Gour, Phys. Rev. D 66, 104022 (2002); O. Dreyer, Phys. Rev. Lett. 90, 081301 (2003); M. Maggiore, Phys. Rev. 100, 141301 (2008); G. Dvali and C. Gomez, Fortschr. Phys. 59, 579 (2011); A. Davidson, Int. J. Mod. Phys. D 23, 1450041 (2014); D. Oriti, D. Pranzetti, and L. Sindoni, Phys. Rev. Lett. 116, 211301 (2016); A. Davidson, Phys. Rev. D 100, 081502(R) (2019).

[21] C. V. Johnson, arXiv:1907.05883 [hep-th]; C. V. Johnson, Class. Quantum Grav. 37, 054003 (2020).

[22] B. P. Dolan, Class. Quantum Grav. 28, 125020 (2011).

[23] B. P. Dolan, Class. Quantum Grav. 28, 235017 (2011).

[24] M. Cvetic, G. W. Gibbons, D. Kubiznak and C. N. Pope, Phys. Rev. D 84, 024037 (2011).

[25] B. P. Dolan, arXiv:1209.1272 (2012).

[26] D. Kubiznak, R. B. Mann, Can. J. Phys., 93999 (2015).

[27] R. B. Mann, The Chemistry of Black Holes. In: Nicolini P., Kaminski M., Mureika J., Bleicher M. (eds), 1st Karl Schwarzschild Meeting on Gravitational Physics. Springer Proceedings in Physics, 170. Springer (2016). 
[28] B. P. Dolan, Mod. Phys. Lett. A 30, 154002 (2015).

[29] T. Padmanabhan, Class. Quantum Grav. 19, 5387 (2002).

[30] T. Padmanabhan and D. Kothawala, Phys. Rept. 531, 115 (2013).

[31] S. Chakraborty and T. Padmanabhan, Phys . Rev. D 42, 104011 (2015).

[32] D. Hansen, D. Kubiznak and R. B. Mann, Class. Quantum Grav. 33, 165005 (2016).

[33] N. Altamirano, D. Kubiznak, R. B. Mann and Z. Sherkatghanad, Galaxies 2, 89 (2014).
[34] Y. Tian and X. -N. Wu, Phys. Rev. D 81, 104013 (2010).

[35] E. Verlinde. J. High Energ. Phys. 2011, 29 (2011).

[36] S. A. Hayward, Phys. Rev. D 74, 104013 (2006).

[37] L. Smarr, Phys. Rev. D 7, 289 (1973).

[38] D. L. Wiltshite, M. Visser and S. M. Scott (Eds.), The Kerr Spacetime: Rotating Black Holes in General Relativity, Cambridge University Press (2009). 